\title{
Construcción y fiabilidad de un cuestionario para eva- luar las necesidades familiares de personas con trastorno mental grave.
}

Construction and reliability of a questionnaire to assess the needs of relatives of patients with severe mental disorder.

\author{
Manuel Gómez Beneyto a , Álvaro Ruiz Rodríguez ', María Luz Palacios c , Namdev Freund Llove- \\ ra d, Alberto Fernández Liria ${ }^{\mathrm{e}}$.
}

${ }^{a}$ Psiquiatra CIBERSAM, ${ }^{b}$ Residente de Psicología Clínica. Hospital Universitario Príncipe de Asturias, ${ }^{c}$ Residente de Psicología Clínica. Hospital Universitario Príncipe de Asturias, ${ }^{d}$ Residente de Psiquiatría. Hospital Universitario Príncipe de Asturias, ${ }^{c}$ Psiquiatra. Hospital Universitario Príncipe de Asturias. Universidad de Alcalá

Correspondencia: Alberto Fernández,Liria (afiria@gmail.com)

Recibido: 15/05/2011; aceptado con modificaciones: 15/02/2012

\begin{abstract}
RESUMEN: Numerosos estudios señalan la importante carga a la que se ven sometidos los familiares de personas con trastorno mental grave, así como el papel fundamental que adquieren en su evolución. En este contexto adquiere una relevancia fundamental el conocimiento de las necesidades no satisfechas que presenta el entorno familiar.

Se presenta el Cuestionario de Necesidades de Familiares de personas con trastornos mentales graves (CNF-TMG) diseñado para identificar tanto las necesidades sentidas y no sentidas por los cuidadores como aquellas que han sido o no han sido satisfechas.

El cuestionario consta de 27 ítems con tres alternativas de respuesta e incluye preguntas relativas a las posibles causas de insatisfacción. Se aplicó a una muestra formada por 77 familiares y dos meses después se llevó a cabo una aplicación retest sobre 55 familiares. Los resultados mostraron una consistencia interna satisfactoria y una estabilidad test-retest razonable. Entre las ventajas aportadas por el CNF-TMG destacan su posibilidad de aplicación a los Planes Integrales de Atención (PIA) y su capacidad para detectar necesidades en las áreas del apoyo personal y de la participación, que hasta ahora no habían sido tomadas en consideración.

PALABRAS CLAVE: evaluación de necesidades, cuidadores, trastornos mentales, cuestionarios, indicadores de salud, apoyo social.
\end{abstract}

ABSTRACT: Several studies report the significant burden born by relatives of people with severe mental illness and the role it plays in the course of the disorder.

We present a questionnaire (CNF-TMG) designed to identify both the needs felt (and not felt) by caregivers and those which are (and are not) met. The questionnaire contains 27 items with three alternative answers as well as questions related to the lack of satisfaction. It was administered to a sample of 77 relatives of patients with severe mental disorder and again two months later. The results showed satisfactory internal consistency and a reasonable test-retest stability.

Among the advantages provided by the CNF-TMG are its applicability to Comprehensive Care Plans (IAP) and its ability to identify needs in the areas of personal support and participation, which until now have not been taken into consideration.

KEY WORDS: needs assessment, caregivers, mental disorders, questionnaires, health status indicators, social support. 
ORIGINALES Y REVISIONES

\section{Introducción}

Los familiares de las personas con trastorno metal grave sufren una pesada carga y, como consecuencia, tienen necesidades cuya satisfacción es importante por derecho propio y además porque la interacción con los familiares es determinante de la evolución de estos trastornos (1).

El reconocimiento de este derecho de los familiares y de la importancia de su colaboración es imprescindible para que, desde los servicios de salud mental pueda llevarse a cabo un plan que debe ser acordado con paciente y los cuidadores informales, centrado tanto en las necesidades de atención médica y psicológica, como en las de participación y de información y que debería facilitar el acceso a la asociación de familiares y usuarios más cercana a su domicilio. Semejante plan debería ser revisado, al menos, anualmente y debería estar incluido claramente en la cartera de servicios.

Las dificultades en la relación entre los profesionales y los cuidadores se han justificado en ocasiones por la ambigüedad en torno a la regulación de la confidencialidad (2). Rapaport (3), en una encuesta de usuarios de servicios, cuidadores y profesionales, encontró que en cada grupo había distintos puntos de vista acerca de qué información debía ser compartida. No existen directrices claras sobre el intercambio de información a pesar de las políticas sobre el rol y los derechos de los cuidadores. Este problema debe ser abordado como una cuestión de urgencia debido a la frustración que con frecuencia se produce en los cuidadores como consecuencia de la falta de servicios para satisfacer sus necesidades(2).

La evaluación de necesidades tanto nivel individual como comunitario, se considera hoy en un elemento central para la organización de la asistencia a las personas con trastorno mental grave (4-7).

Aunque los conceptos de "necesidad" y "evaluación” de la misma están aún sujetos a discusión (5), se han desarrollado varios instrumentos para evaluar las necesidades de las personas con trastorno metal grave $(5,8,9)$. Alguno de ellos, como el CAN (9) se han incorporado a la práctica estándar. La detección de las necesidades no satisfechas permite planificar intervenciones destinadas a satisfacerlas o a remover las barreras que impiden su satisfacción y permite evaluar la capacidad de estas intervenciones para resolverlas $(10,11)$.

En nuestro país, en el documento de consenso "Modelo de atención a las personas con enfermedad mental grave" (12), se señala que las necesidades más frecuentes percibidas por los familiares son: disponer de información adecuada sobre la enfermedad, ser tenidos en cuenta en la toma de decisiones que puedan repercutir en la familia, ayuda material y prestaciones económicas, periodos de descanso o respiro, apoyo emocional y apoyo técnico. Se explicita además la necesidad de aportar una respuesta a estas necesidades por parte de los organismos implicados y 
ORIGINALES Y REVISIONES

tal y como está incluido en los Planes de Continuidad de Cuidados, con el objetivo de reforzar las redes naturales de inserción social de las personas con enfermedad mental grave. Se ofrecen una serie de propuestas para llevar esto a cabo aunque no se define claramente el modo de medir y operativizar estas necesidades.

Los instrumentos que se han propuesto para medir las necesidades, sin embargo, presentan algunas limitaciones. Algunos se centran sólo en las necesidades fundamentalmente de información que servirían para orientar las intervenciones psicoterapéuticas familiares (13-16). Otros miden más bien el grado de satisfacción con la atención recibida o las políticas asistenciales $(17,18)$ o la carga que representa convivir con una persona que padece un TMG (18-20). Otros focalizan en un aspecto parcial, como es el caso del cuestionario FAD de Epstein, Baldwin y Bishop (21), que valora habilidades de los familiares para ofrecer apoyo mutuo o bien otro que se centra en la necesidad de liberarse temporalmente de la tarea de cuidar (22). El ABC, cuya validación fue publicada durante la realización de nuestro estudio (18), tiene un alcance parecido al que proponemos, aunque no permite saber si las necesidades insatisfechas está insatisfechas porque no han sido percibidas o porque habiéndolo sido, no han sido resueltas satisfactoriamente.

Para identificar potenciales áreas de intervención no cubiertas, en primer lugar, parece imprescindible inspeccionar el entorno natural preguntando directamente a los familiares que convivan habitualmente con el enfermo $(18,23)$.

El trabajo que presentamos forma parte del proyecto NEUFAM que pretende identificar y describir las necesidades asistenciales no satisfechas de las personas en tratamiento en los servicios públicos de salud mental españoles por un trastorno mental grave esquizofrénico (TMGE) y las de sus cuidadores, e identificar las causas de la falta de satisfacción de esas necesidades

Como parte de ese proyecto, intentamos desarrollar un instrumento capaz de detectar necesidades satisfechas o no satisfechas percibidas por los cuidadores de las personas con trastorno mental grave. Tal instrumento debería servir para un doble propósito. En primer lugar debería ser capaz de determinar si una determinada necesidad ha sido sentida o no por el cuidador y, caso de haber sido sentida, si ha sido satisfecha. Además, el instrumento debería ser capaz de servir también para evaluar si las intervenciones propuestas para atender las necesidades detectadas han sido eficaces.

En la medida en la que, además de servir para la investigación en curso, pretende servir para facilitar la exploración de las necesidades de los familiares en la práctica asistencial estándar, debería ser de aplicación sencilla y rápida. 
ORIGINALES Y REVISIONES

\section{Material y método}

Con la finalidad de obtener un inventario de necesidades de familiares de pacientes con trastornos mentales graves, dos psiquiatras con amplia experiencia clínica elaboraron un primer borrador basado en cuestionarios ya publicados (13-17, 24) y en su propia experiencia. Este primer borrador fue revisado y ampliado por familiares de enfermos mentales miembros de órganos de gobierno de FEAFES y grupos de familiares de dos asociaciones, así como por técnicos de la Federación, dando como resultado un segundo listado de 44 ítems.

Tras eliminar repeticiones y solapamientos los 44 ítems fueron finalmente sintetizados y concretados en 27 preguntas, agrupables en cuatro áreas de necesidades: "conocimientos/información", "apoyo instrumental", "apoyo personal" y "participación".

Para cada una de las preguntas se preparó una escala de respuestas con tres alternativas: "he sentido esta necesidad y me la han satisfecho", "he sentido esta necesidad pero no me la han satisfecho", "no he sentido esta necesidad".

Al final del cuestionario se añadieron cuatro preguntas sobre las posibles causas de insatisfacción referidas a los profesionales o al propio familiar y no a otros aspectos del proceso de cuidado o de la estructura de los servicios: "los profesionales con los que me he relacionado no tienen la costumbre de dar explicaciones a la familia", "no he tenido interés en hablar con los profesionales", "no creo que puedan aportarme nada útil, no he preguntado nada a los profesionales ya que ellos están para ayudar solamente a los pacientes y no a los familiares" y "he preguntado a los profesionales pero nunca me han dado explicaciones claras".

Una vez comprobada la comprensibilidad del cuestionario se administró a una muestra de familiares de enfermos mentales atendidos en dos centros de rehabilitación psicosocial de Madrid. Los pacientes fueron 46 hombres y 31 mujeres, con una edad media de 37.4 años y una duración de enfermedad de 13.5 años. Se eligieron familiares que habitualmente se ocupaban del paciente. Cuando el paciente convivía con más de un familiar se eligió el familiar "clave". En su mayoría (87\%) fueron los padres, que vivían con el paciente y que le atendían a diario. Los test fueron administrados por residentes de Psicología Clínica y Psiquiatría del Hospital Universitario Príncipe de Asturias, siendo la duración media de aplicación de aproximadamente 35 minutos.

Con el fin de determinar la fiabilidad test-retest se volvió a administrar el cuestionario a 55 de ellos tras un intervalo de dos meses. 


\section{Resultados}

\section{En la tabla 1 se presentan los resultados de la fiabilidad test-retest medi- da mediante el coeficiente V de Crammer pregunta a pregunta. Solamente cuatro}

Tabla 1

Fiabilidad Test-Retest.

\begin{tabular}{|c|c|c|c|}
\hline & $\mathbf{v C}$ & $\mathbf{p}$ & $\mathbf{n}$ \\
\hline $\begin{array}{l}\text { Cómo encarar las crisis causadas por la enfermedad (amenaza de suicidio, agitación, violencia, etc.) } \\
\text { de mi familiar enfermo. }\end{array}$ & 0.39 & 0.004 & 53 \\
\hline $\begin{array}{l}\text { Cómo reaccionar ante las conductas poco razonables de mi familiar enfermo (los delirios, las } \\
\text { alucinaciones, el abandono, la negligencia) }\end{array}$ & 0.39 & 0.003 & 53 \\
\hline $\begin{array}{l}\text { Conocer la causa, el diagnóstico, los síntomas y el pronóstico de la enfermedad que padece mi } \\
\text { familiar. }\end{array}$ & 0.35 & 0.17 & 53 \\
\hline Saber si la enfermedad que padece mi familiar enfermo es hereditaria & 0.35 & 0.17 & 51 \\
\hline Saber para que sirve la medicación y cuales son sus efectos secundarios. & 0.56 & 0.002 & 53 \\
\hline $\begin{array}{l}\text { Saber qué hacer para evitar que se produzca un abandono de la medicación y cómo reaccionar si se } \\
\text { produce. }\end{array}$ & 0.66 & 0.000 & 49 \\
\hline Saber que es la psicoterapia y para que sirve & 0.41 & 0.07 & 52 \\
\hline Saber que es la rehabilitación y para que sirve & 0.18 & 0.8 & 52 \\
\hline $\begin{array}{l}\text { Cómo será la vida de mi familiar enfermo en el futuro (si podrá llevar una vida independiente, si } \\
\text { podrá formar o mantener una familia propia). }\end{array}$ & 0.53 & 0.006 & 52 \\
\hline $\begin{array}{l}\text { Estar informado de aspectos legales, jurídicos y administrativos relacionados con la enfermedad de } \\
\text { mi familiar }\end{array}$ & 0.38 & 0.005 & 51 \\
\hline $\begin{array}{l}\text { Tener información sobre los servicios disponibles para ayudarme como cuidador cuando mi familiar } \\
\text { enfermo tenga que ingresar urgentemente y en contra de su voluntad en el hospital }\end{array}$ & 0.45 & 0.03 & 52 \\
\hline $\begin{array}{l}\text { Tener información sobre los servicios disponibles para ayudarme como cuidador cuando mi familiar } \\
\text { enfermo resida en casa }\end{array}$ & 0.52 & 0.007 & 52 \\
\hline $\begin{array}{l}\text { Saber cómo compensar los gastos relacionados con el cuidado de mi familiar (prestaciones } \\
\text { económicas, becas, descuentos, etc). }\end{array}$ & 0.57 & 0.003 & 50 \\
\hline Estar informado de cómo y dónde presentar una queja si no estoy conforme con el tratamiento. & 0.71 & 0.000 & 52 \\
\hline Estar informado de cómo y dónde presentar una queja en caso de discriminación y acoso. & 0.46 & 0.03 & 52 \\
\hline Recibir información sobre el movimiento asociativo y los servicios que presta a los cuidadores & 0.27 & 0.44 & 52 \\
\hline $\begin{array}{l}\text { Consultar con un profesional (psiquiatra, psicólogo, enfermero) a causa del estrés que me causa } \\
\text { cuidar de mi familiar }\end{array}$ & 0.58 & 0.002 & 51 \\
\hline $\begin{array}{l}\text { Que los profesionales me escuchen y que cuenten con mi opinión a la hora de evaluar a mi familiar } \\
\text { enfermo }\end{array}$ & 0.25 & 0.53 & 50 \\
\hline $\begin{array}{l}\text { Que los profesionales me escuchen y que cuenten con mi opinión cuando advierto sobre los "síntomas } \\
\text { de alarma" }\end{array}$ & 0.40 & 0.08 & 51 \\
\hline $\begin{array}{l}\text { Que los profesionales me escuchen y que cuenten con mi opinión para planificar el tratamiento y la } \\
\text { rehabilitación de mi familiar }\end{array}$ & 0.41 & 0.06 & 51 \\
\hline Que los profesionales me hablen con palabras que yo pueda comprender y en mi propio idioma & 0.50 & 0.01 & 51 \\
\hline $\begin{array}{l}\text { Que los profesionales consideren el derecho de la familia a no asumir responsabilidades que no le } \\
\text { corresponden }\end{array}$ & 0.58 & 0.003 & 47 \\
\hline Recibir ayuda económica para compensar los gastos generados por la enfermedad de mi familiar & 0.62 & 0.001 & 51 \\
\hline Recibir ayuda para sustituirme en el cuidado de mi familiar enfermo cuando yo no pueda hacerlo & 0.41 & 0.07 & 49 \\
\hline Recibir atención especializada, especialmente programas de intervención familiar. & 0.44 & 0.04 & 50 \\
\hline Necesidad de sentir la compañía de otras personas que tengan el mismo problema que yo & 0.55 & 0.004 & 52 \\
\hline $\begin{array}{l}\begin{array}{l}\text { Necesidad de sentir la solidaridad de la gente, que me respeten, que me apoyen y que comprendan mi } \\
\text { situación }\end{array} \\
\end{array}$ & 0.43 & 0.04 & 51 \\
\hline $\begin{array}{l}\text { Necesidad de descansar y de reducir la sensación de responsabilidad en el cuidado de mi familiar } \\
\text { enfermo }\end{array}$ & 0.68 & 0.000 & 51 \\
\hline Media & 44 & & \\
\hline
\end{tabular}


preguntas obtienen un coeficiente inaceptable: "Conocer la causa, el diagnóstico, los síntomas ...", "Saber si la enfermedad que padece mi familiar es hereditaria", "Recibir información sobre el movimiento asociativo" y "Que los profesionales me escuchen ...". La consistencia interna del cuestionario medida con el alfa de Cronbach es de 0.80 .

En las tabla 2 se observan los resultados agrupados por áreas de necesidad y el total de respuestas válidas. El 54\% de las respuestas válidas correspondieron a necesidades no satisfechas, incluyendo las no sentidas. Entre las necesidades no sentidas destacan "Estar informado de cómo y dónde presentar una queja en caso de discriminación y acoso" (73\%) y "Estar informado de cómo y dónde presentar una queja si no estoy conforme con el tratamiento" (64\%), seguidas de "Recibir ayuda para sustituirme en el cuidado de mi familiar enfermo cuando yo no pueda hacerlo" (55\%), "Que los profesionales consideren el derecho de la familia a no asumir responsabilidades que no le corresponden" (53\%). En cuanto a necesidades sentidas pero no satisfechas destaca "Cómo será la vida de mi familiar enfermo en el futuro (si podrá llevar una vida independiente, si podrá formar o mantener una familia propia) (51\%).

Tabla 2

Respuestas válidas a las preguntas según percepción de la necesidad y satisfacción.

\begin{tabular}{|l|c|c|c|c|}
\hline Preguntas & $\begin{array}{c}\text { He sentido esta } \\
\text { necesidad y me } \\
\text { la han satisfecho }\end{array}$ & $\begin{array}{c}\text { He sentido esta } \\
\text { necesidad pero } \\
\text { no } \mathbf{m e ~ l a ~ h a n ~} \\
\text { satisfecho }\end{array}$ & $\begin{array}{c}\text { No he sentido } \\
\text { esta necesidad }\end{array}$ & TOTALES \\
\hline $\begin{array}{l}\text { Cómo encarar las crisis causadas por la enfermedad } \\
\text { (amenaza de suicidio, agitación, violencia, etc.) de mi } \\
\text { familiar enfermo. }\end{array}$ & $54(4)$ & $26(20)$ & $21(16)$ & $100(77)$ \\
\hline $\begin{array}{l}\text { Cómo reaccionar ante las conductas poco } \\
\text { razonables de mi familiar enfermo (los delirios, las } \\
\text { alucinaciones, el abandono, la negligencia) }\end{array}$ & $50(38)$ & $36(27)$ & $14(11)$ & $100(76)$ \\
\hline $\begin{array}{l}\text { Conocer la causa, el diagnóstico, los síntomas y el } \\
\text { pronóstico de la enfermedad que padece mi familiar. }\end{array}$ & $74(57)$ & $24(18)$ & $3(2)$ & $100(77)$ \\
\hline $\begin{array}{l}\text { Saber si la enfermedad que padece mi familiar } \\
\text { enfermo es hereditaria }\end{array}$ & $51(38)$ & $23(17)$ & $26(19)$ & $100(72)$ \\
\hline $\begin{array}{l}\text { Saber para qué sirve la medicación y cuáles son sus } \\
\text { efectos secundarios. }\end{array}$ & $63(47)$ & $29(22)$ & $8(6)$ & $100(75)$ \\
\hline $\begin{array}{l}\text { Saber qué hacer para evitar que se produzca un } \\
\text { abandono de la medicación y cómo reaccionar si se } \\
\text { produce. }\end{array}$ & $48(34)$ & $14(10)$ & $38(27)$ & $100(71)$ \\
\hline
\end{tabular}




\section{ORIGINALES Y REVISIONES}

\begin{tabular}{|c|c|c|c|c|}
\hline Saber que es la psicoterapia y para qué sirve & $57(42)$ & $29(21)$ & $14(10)$ & $100(73)$ \\
\hline Saber que es la rehabilitación y para qué sirve & $72(54)$ & $21(16)$ & $7(5)$ & $100(75)$ \\
\hline $\begin{array}{l}\text { Cómo será la vida de mi familiar enfermo en el } \\
\text { futuro (si podrá llevar una vida independiente, si } \\
\text { podrá formar o mantener una familia propia). }\end{array}$ & $35(26)$ & $51(38)$ & $14(10)$ & $100(74)$ \\
\hline $\begin{array}{l}\text { Estar informado de aspectos legales, jurídicos y } \\
\text { administrativos relacionados con la enfermedad de } \\
\text { mi familiar }\end{array}$ & $47(35)$ & $27(20)$ & $26(19)$ & $100(74)$ \\
\hline $\begin{array}{l}\text { Tener información sobre los servicios disponibles } \\
\text { para ayudarme como cuidador cuando mi familiar } \\
\text { enfermo tenga que ingresar urgentemente y en contra } \\
\text { de su voluntad en el hospital }\end{array}$ & $43(32)$ & $27(20)$ & $30(23)$ & $100(75)$ \\
\hline $\begin{array}{l}\text { Tener información sobre los servicios disponibles } \\
\text { para ayudarme como cuidador cuando mi familiar } \\
\text { enfermo resida en casa }\end{array}$ & $47(36)$ & $28(21)$ & $25(19)$ & $100(76)$ \\
\hline $\begin{array}{l}\text { Saber cómo compensar los gastos relacionados con } \\
\text { el cuidado de mi familiar (prestaciones económicas, } \\
\text { becas, descuentos, etc). }\end{array}$ & $45(33)$ & $42(31)$ & $13(10)$ & $100(74)$ \\
\hline $\begin{array}{l}\text { Recibir ayuda para sustituirme en el cuidado de mi } \\
\text { familiar enfermo cuando yo no pueda hacerlo }\end{array}$ & $15(11)$ & $30(22)$ & $55(41)$ & $100(74)$ \\
\hline $\begin{array}{l}\text { Recibir atención especializada, especialmente } \\
\text { programas de intervención familiar. }\end{array}$ & $49(37)$ & $20(15)$ & $31(23)$ & $100(75)$ \\
\hline $\begin{array}{l}\text { Necesidad de sentir la compañía de otras personas } \\
\text { que tengan el mismo problema que yo }\end{array}$ & $50(39)$ & $15(12)$ & $35(27)$ & $100(78)$ \\
\hline $\begin{array}{l}\text { Necesidad de sentir la solidaridad de la gente, que } \\
\text { me respeten, que me apoyen y que comprendan mi } \\
\text { situación }\end{array}$ & $45(34)$ & $19(14)$ & $36(27)$ & $100(72)$ \\
\hline $\begin{array}{l}\text { Necesidad de descansar y de reducir la sensación de } \\
\text { responsabilidad en el cuidado de mi familiar enfermo }\end{array}$ & $21(16)$ & $37(28)$ & $42(32)$ & $100(76)$ \\
\hline $\begin{array}{l}\text { Consultar con un profesional (psiquiatra, psicólogo, } \\
\text { enfermero) a causa del estrés que me causa cuidar de } \\
\text { mi familiar }\end{array}$ & $53(39)$ & $15(11)$ & $32(23)$ & $100(73)$ \\
\hline $\begin{array}{l}\text { Que los profesionales consideren el derecho de la } \\
\text { familia a no asumir responsabilidades que no le } \\
\text { corresponden }\end{array}$ & $26(19)$ & $21(15)$ & $53(39)$ & $100(73)$ \\
\hline $\begin{array}{l}\text { Que los profesionales me hablen con palabras que yo } \\
\text { pueda comprender y en mi propio idioma }\end{array}$ & $73(56)$ & $9(7)$ & $18(14)$ & $100(77)$ \\
\hline $\begin{array}{l}\text { Estar informado de cómo y dónde presentar una } \\
\text { queja si no estoy conforme con el tratamiento. }\end{array}$ & $16(12)$ & $20(15)$ & $64(47)$ & $100(74)$ \\
\hline $\begin{array}{l}\text { Estar informado de cómo y dónde presentar una } \\
\text { queja en caso de discriminación y acoso. }\end{array}$ & $12(9)$ & $15(11)$ & $73(55)$ & $100(75)$ \\
\hline $\begin{array}{l}\text { Recibir información sobre el movimiento asociativo } \\
\text { y los servicios que presta a los cuidadores }\end{array}$ & $38(28)$ & $28(21)$ & $34(25)$ & $100(74)$ \\
\hline $\begin{array}{l}\text { Que los profesionales me escuchen y que cuenten con } \\
\text { mi opinión a la hora de evaluar a mi familiar enfermo }\end{array}$ & $87(65)$ & $8(6)$ & $5(4)$ & $100(75)$ \\
\hline $\begin{array}{l}\text { Que los profesionales me escuchen y que cuenten } \\
\text { con mi opinión cuando advierto sobre los "síntomas } \\
\text { de alarma" }\end{array}$ & $64(49)$ & $11(8)$ & $25(19)$ & $100(76)$ \\
\hline $\begin{array}{l}\text { Que los profesionales me escuchen y que cuenten } \\
\text { con mi opinión para planificar el tratamiento y la } \\
\text { rehabilitación de mi familiar }\end{array}$ & $75(57)$ & $13(10)$ & $12(9)$ & $100(76)$ \\
\hline Total de preguntas & $46(842)$ & $23(424)$ & $31(581)$ & $100(1847)$ \\
\hline
\end{tabular}


Entre las necesidades sentidas pero no satisfechas destacan "Cómo será la vida de mi familiar enfermo en el futuro (si podrá llevar una vida independiente, si podrá formar o mantener una familia propia)" (51\%) y "Saber cómo compensar los gastos relacionados con el cuidado de mi familiar (prestaciones económicas, becas, descuentos, etc)" (42\%), seguidas por "Necesidad de descansar y de reducir la sensación de responsabilidad en el cuidado de mi familiar enfermo" (37\%) y por "Cómo reaccionar ante las conductas poco razonables de mi familiar enfermo (los delirios, las alucinaciones, el abandono, la negligencia)" (36\%).

En la Tabla 3 se describen los motivos de insatisfacción. El motivo que se presenta con más frecuencia es "No he pedido nada a los profesionales ya que ellos están para ayudar solamente a los pacientes y no a los familiares" seguido de "He preguntado a los profesionales pero nunca me han dado explicaciones claras".

Tabla 3

Porcentajes de familiares según motivos de insatisfacción en las necesidades sentidas.

\begin{tabular}{|l|c|}
\hline & $\%(\mathrm{n})$ \\
\hline Los profesionales con los que me he relacionado no tienen la costumbre de dar explicaciones a la familia & $25(12)$ \\
\hline No he tenido interés en hablar con los profesionales, no creo que puedan aportarme nada útil & $13(6)$ \\
\hline $\begin{array}{l}\text { No he pedido nada a los profesionales ya que ellos están para ayudar solamente a los pacientes y no a los } \\
\text { familiares }\end{array}$ & $33(16)$ \\
\hline He preguntado a los profesionales pero nunca me han dado explicaciones claras & $29(14)$ \\
\hline
\end{tabular}

\section{Discusión}

El análisis psicométrico de la fiabilidad del CNF-TMG aplicado en dos centros de rehabilitación psicosocial de la Comunidad de Madrid muestra un nivel de consistencia interna satisfactorio, lo que indica que se trata de instrumento cuyos ítems se comportan de manera homogénea. La prueba test-retest expresada con el coeficiente $\mathrm{V}$ de Crammer indica una estabilidad razonable, si bien podría mejorarse suprimiendo los cuatro ítems que presentan un coeficiente claramente no significativo. Debe tenerse en cuenta que la estabilidad de la medida también depende de factores situacionales externos al cuestionario, como bien pudiera ser el grado de estabilidad de la patología del familiar y de otras circunstancias socio-familiares.

El presente estudio adolece de varias limitaciones. Desde el punto de vista metodológico habría sido deseable obtener la información inicial de forma más sistemática y quizás mediante el empleo de grupos focales de familiares, 
ORIGINALES Y REVISIONES

tal como se ha llevado a cabo en otros estudios con grupos de usuarios (25). Es posible que de esta forma se hubiese llegado a extraer información más cercana a las necesidades reales de los familiares, menos contaminada por la perspectiva de los profesionales que redactaron el inventario inicial de necesidades.

Por otra parte la ausencia de correlación significativa en varias preguntas del cuestionario en la prueba de test-retest puede indicar que las preguntas estaban formuladas con ambigüedades, lo cual podría explicar la diferencia entre las respuestas dadas en la primera y la segunda administración. Sin embargo, si nos fijamos en ellas, son perfectamente comprensibles, por ejemplo "Saber que es la rehabilitación y para qué sirve" o bien "Recibir información sobre el movimiento asociativo y los servicios que presta a los cuidadores".

El CNF-TMG aporta dos novedades importantes respecto a otros cuestionarios utilizados para evaluar las necesidades de los familiares de las personas con TMG. En primer lugar, permite conocer las necesidades sentidas por los familiares y las necesidades que se encuentran satisfechas. Las primeras son la suma de "He sentido esta necesidad y me la han satisfecho" y "He sentido esta necesidad pero no me la han satisfecho", lo que configura la mayor parte de los casos. Un objetivo prioritario en los cuestionarios revisados tienen por objeto evaluar la eficacia de las intervenciones familiares de naturaleza psicoeducativa $(13,16)$, mientras que el CNF-TMG puede ser utilizado además para detectar las necesidades familiares no satisfechas en el contexto de la atención a las personas con trastorno mental grave mediante Planes Integrados de Atención (PIA). De la misma forma que en los PIA se identifican las necesidades de los pacientes y se hace un plan de actuación para satisfacerlas, parece razonable hacer lo mismo con las necesidades de los familiares que cuidan del paciente, bien como una parte del PIA o como un PIA específico para el familiar. Puede resultar útil para plantear objetivos de intervención con familiares diferenciar si las necesidades evaluadas están o no satisfechas, especificando además si la necesidad está satisfecha mediante la participación en los PIA del SNS o por no haberla sentido.

En segundo lugar, el CNF-TMG detecta necesidades en dos áreas importantes que hasta ahora no han sido tomadas en consideración, que son el apoyo personal y la participación. El área de apoyo personal la componen ítems referentes a necesidades no contempladas habitualmente en las intervenciones terapéuticas y para las cuales no tenemos respuestas adecuadas, tales como necesidad de compañía y de sentir la solidaridad de otras familias que comparten el mismo problema, la necesidad de descansar y de reducir la sensación de responsabilidad y de recibir tratamiento en caso necesario. El movimiento de "la recuperación" y "el empowerment" ha hecho visibles necesidades "existenciales" que hasta ahora no han sido reconocidas. Aunque estas necesidades son de carácter universal en el caso de las personas con TMG se encuentran particularmente afectadas 
por el estigma y la discriminación (26). Su satisfacción requiere reforzar la red de apoyo social, facilitar el desarrollo de grupos de ayuda mutua y promover el asociacionismo (27), ofertar alojamientos temporales para los pacientes con el fin de proporcionar descanso a los familiares cuidadores (22), aplicar terapias específicamente orientadas a reducir el auto-estigma del familiar (28), pero también la adopción de una actitud empática y respetuosa.

Las necesidades de participación son objeto también de atención en el discurso actual de la rehabilitación de las personas con trastornos mentales graves, pero las encuestas indican que su participación no es real y efectiva si no solamente simbólica, al menos en nuestro país. Los profesionales de salud mental miembros de la Asociación Española de Neuropsiquiatría que fueron consultados mediante una encuesta confirmaron que la participación de los usuarios y familiares en el Plan Inividualizado de Atención es escasa, la participación de las asociaciones en la planificación y evaluación de servicios es casi nula y en la formación del personal y en investigación nula. Sin embargo la actitud es favorable a incrementar la participación, especialmente a nivel individual (27).

El patrón global de áreas de necesidad detectadas mediante el presente estudio normativo con el que se presenta el CNF-TMG, indica la existencia de mayor grado de necesidades satisfechas en el área de conocimientos (63\%) y mayor grado de ausencia de necesidad en las áreas de apoyo personal y participación (81\% y $84 \%$ respectivamente, si reunimos los porcentajes relativos a las necesidades satisfechas y las no sentidas en la actualidad). Por su parte, la mayor proporción de necesidades sentidas y no satisfechas se sitúa en el área del apoyo instrumental $(30 \%)$. Este patrón de resultados contrasta con el obtenido recientemente en otros países occidentales, donde la necesidad de sentir la solidaridad de la gente parece ser una demanda más firme (2) relacionan la mayor solicitud de recursos instrumentales con la carencia de recursos del país . Resulta lógico pensar que la demanda de solidaridad y apoyo social pueda surgir una vez se encuentren suficientemente satisfechas aquellas necesidades que comportan mayor carga objetiva. Alternativamente, en nuestra comunidad es posible que la persistencia de demandas de apoyo instrumental esté más relacionada con la necesidad de reestructurar los recursos humanos disponibles, primando más la ratio de profesionales dedicados a la puesta en funcionamiento de los PIA (trabajadores sociales, educadores, terapeutas ocupacionales, enfermeros especializados, etc.) frente a la predominancia actual de profesionales que programan y guían el tratamiento (psiquiatras y psicólogos).

De cara al planteamiento de futuros trabajos, consideramos importante prestar atención a la necesidad de los familiares de obtener información relativa al "futuro" de los pacientes (ítem: "Cómo será la vida de mi familiar enfermo en el futuro (si podrá llevar una vida independiente, si podrá formar o mantener 
ORIGINALES Y REVISIONES

una familia propia)"). En el presente estudio es la única necesidad no satisfecha que obtiene un porcentaje superior al 50\%. Se trata de una necesidad detectada de forma recurrente en estudios previos, con índices de relevancia elevados (15, $18,20)$ y llegando a alcanzar índices de demanda del $94.3 \%$ (27). Estos resultados podrían indicar que se trata de una necesidad de mayor alcance de lo que pretendía inicialmente este estudio, por lo que proponemos profundizar en ella en investigaciones ulteriores. Para indagar en su significado podría subdividirse en varias cuestiones psicoeducaivas diferentes (p. ej. potenciales evoluciones de la patología, planificación de cuidados futuros, potenciales recursos asistenciales que garanticen la sostenibilidad del enfermo en ausencia del familiar, recursos tutelares y otros mediadores profesionales sustitutorios, efectos a largo plazo del tratamiento farmacológico, alternativas ocupacionales, etc.). Su estudio en la literatura ha sido limitado hasta la actualidad $(29,30)$

De cara a la valoración integral de las necesidades de familiares, otras áreas complementarias a las que atender podrían ser la salud del familiar, el estilo de afrontamiento, la red grupal de apoyo en el entorno inmediato y la satisfacción con el cuidado $(27,31)$. Se trata de aspectos relevantes en la detección de necesidades que podrían incluirse en planes de intervención más exhaustivos, valorándose mediante técnicas de exploración diferentes a los cuestionarios de autoinforme. 
Anexo 1

\section{CUESTIONARIO SOBRE LAS NECESIDADES DE LAS PERSONAS QUE CUIDAN A UN FAMILIAR QUE PADECE UNA ENFERMEDAD MENTAL.}

¿Vive usted con un familiar enfermo mental? SI NO

¿Qué edad tiene su familiar? $\quad 0$ - $25 \quad 25-50 \quad 50-65 \quad$ Más de 65

¿Cuántos años lleva su familiar enfermo?

¿Cual es el sexo de su familiar? Hombre Mujer

¿Que relación tiene con el? Madre/padre Hermano/hermana Otra

¿Con que frecuencia atiende a las necesidades de su familiar?

$\begin{array}{llll}\text { Todos los } & \text { Casi todos } & \text { Una vez por } & \text { Menos de una } \\ \text { días } & \text { los días } & \text { semana } & \text { vez por semana }\end{array}$

A continuación encontrará unas preguntas sobre las necesidades que usted puede haber sentido en relación con el cuidado de su familiar enfermo. Contéstelas rodeando con un círculo la respuesta que mejor refleje su opinión.

1. Cómo encarar las crisis causadas por la enfermedad (amenaza de suicidio, agitación, violencia, etc.) de mi familiar enfermo.

\begin{tabular}{l|l|lll|} 
HE SENTIDO ESTA NECESIDAD & HE SENTIDO ESTA NECESIDAD & NO HE SENTIDO \\
Y ME LA HAN SATISFECHO & PERO NO ME LA HAN SATISFECHO & ESTA NECESIDAD \\
\hline
\end{tabular}


ORIGINALES Y REVISIONES

2. Cómo reaccionar ante las conductas poco razonables de mi familiar enfermo (los delirios, las alucinaciones, el abandono, la negligencia)

HE SENTIDO ESTA NECESIDAD Y ME LA HAN SATISFECHO
HE SENTIDO ESTA NECESIDAD PERO NO ME LA HAN SATISFECHO
NO HE SENTIDO ESTA NECESIDAD

3. Conocer la causa, el diagnóstico, los síntomas y el pronóstico de la enfermedad que padece mi familiar.

HE SENTIDO ESTA NECESIDAD

HE SENTIDO ESTA NECESIDAD

NO HE SENTIDO

Y ME LA HAN SATISFECHO

PERO NO ME LA HAN SATISFECHO

ESTA NECESIDAD

4. Saber si la enfermedad que padece mi familiar enfermo es hereditaria

\begin{tabular}{|l|l|lll|}
\hline HE SENTIDO ESTA NECESIDAD & HE SENTIDO ESTA NECESIDAD & NO HE SENTIDO \\
Y ME LA HAN SATISFECHO & PERO NO ME LA HAN SATISFECHO & ESTA NECESIDAD \\
\hline
\end{tabular}

5. Saber para que sirve la medicación y cuales son sus efectos secundarios.

\begin{tabular}{|l|ll|ll|}
\hline HE SENTIDO ESTA NECESIDAD & HE SENTIDO ESTA NECESIDAD & $\begin{array}{l}\text { NO HE SENTIDO } \\
\text { ESTA NECESIDAD }\end{array}$ \\
\hline
\end{tabular}

6. Saber qué hacer para evitar que se produzca un abandono de la medicación y cómo reaccionar si se produce.

\begin{tabular}{l|l|l|l|l|} 
HE SENTIDO ESTA NECESIDAD & HE SENTIDO ESTA NECESIDAD & NO HE & SENTIDO \\
Y ME LA HAN SATISFECHO & PERO NO ME LA HAN SATISFECHO & ESTA NECESIDAD \\
\hline
\end{tabular}

7. Saber que es la psicoterapia y para que sirve

\begin{tabular}{l|l|l|l|l} 
HE SENTIDO ESTA NECESIDAD & HE SENTIDO ESTA NECESIDAD & NO HE SENTIDO \\
Y ME LA HAN SATISFECHO & PERO NO ME LA HAN SATISFECHO & ESTA NECESIDAD \\
\hline
\end{tabular}

8. Saber que es la rehabilitación y para que sirve

HE SENTIDO ESTA NECESIDAD Y ME LA HAN SATISFECHO
HE SENTIDO ESTA NECESIDAD PERO NO ME LA HAN SATISFECHO
NO HE SENTIDO ESTA NECESIDAD 
9. Cómo será la vida de mi familiar enfermo en el futuro (si podrá llevar una vida independiente, si podrá formar o mantener una familia propia).

10. Estar informado de aspectos legales, jurídicos y administrativos relacionados con la enfermedad de mi familiar

HE SENTIDO ESTA NECESIDAD HE SENTIDO ESTA NECESIDAD NO HE SENTIDO Y ME LA HAN SATISFECHO PERO NO ME LA HAN SATISFECHO ESTA NECESIDAD

11. Tener información sobre los servicios disponibles para ayudarme como cuidador cuando mi familiar enfermo tenga que ingresar urgentemente y en contra de su voluntad en el hospital.

\begin{tabular}{l|l|lll|} 
HE SENTIDO ESTA NECESIDAD & HE SENTIDO ESTA NECESIDAD & NO HE SENTIDO \\
Y ME LA HAN SATISFECHO & PERO NO ME LA HAN SATISFECHO & ESTA NECESIDAD \\
\hline
\end{tabular}

12. Tener información sobre los servicios disponibles para ayudarme como cuidador cuando mi familiar enfermo resida en casa

13. Saber cómo compensar los gastos relacionados con el cuidado de mi familiar (prestaciones económicas, becas, descuentos, etc). PERO NO ME LA HAN SATISFECHO ESTA NECESIDAD

14. Estar informado de cómo y dónde presentar una queja si no estoy conforme con el tratamiento.

\begin{tabular}{l|l|lll|} 
HE SENTIDO ESTA NECESIDAD & HE SENTIDO ESTA NECESIDAD & NO HE SENTIDO \\
Y ME LA HAN SATISFECHO & PERO NO ME LA HAN SATISFECHO & ESTA NECESIDAD \\
\hline
\end{tabular}


ORIGINALES Y REVISIONES

15. Estar informado de cómo y dónde presentar una queja en caso de discriminación y acoso.

\begin{tabular}{l|l|l|l|l} 
HE SENTIDO ESTA NECESIDAD & HE SENTIDO ESTA NECESIDAD & NO HE SENTIDO \\
Y ME LA HAN SATISFECHO & PERO NO ME LA HAN SATISFECHO & ESTA NECESIDAD
\end{tabular}

16. Recibir información sobre el movimiento asociativo y los servicios que presta a los cuidadores

\begin{tabular}{|l|llll|lll|} 
HE SENTIDO ESTA NECESIDAD & HE & SENTIDO & ESTA & NECESIDAD & NO & HE & SENTIDO
\end{tabular}

\begin{tabular}{l|l|r} 
Y ME LA HAN SATISFECHO & PERO NO ME LA HAN SATISFECHO & ESTA NECESIDAD
\end{tabular}

17. Consultar con un profesional (psiquiatra, psicólogo, enfermero) a causa del estrés que me causa cuidar de mi familiar

\begin{tabular}{l|l|l|l|} 
HE SENTIDO ESTA NECESIDAD & HE SENTIDO ESTA NECESIDAD & NO HE & SENTIDO \\
Y ME LA HAN SATISFECHO & PERO NO ME LA HAN SATISFECHO & ESTA NECESIDAD \\
\hline
\end{tabular}

18. Que los profesionales me escuchen y que cuenten con mi opinión a la hora de evaluar a mi familiar enfermo

\begin{tabular}{l|l|ll} 
HE SENTIDO ESTA NECESIDAD & HE SENTIDO ESTA NECESIDAD & NO HE SENTIDO \\
Y ME LA HAN SATISFECHO & PERO NO ME LA HAN SATISFECHO & ESTA NECESIDAD \\
\hline
\end{tabular}

19. Que los profesionales me escuchen y que cuenten con mi opinión cuando advierto sobre los "síntomas de alarma"

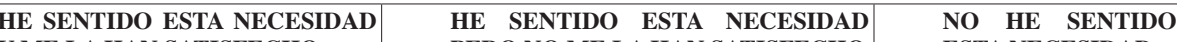
\begin{tabular}{l|l|l|} 
Y ME LA HAN SATISFECHO & PERO NO ME LA HAN SATISFECHO & ESTA NECESIDAD \\
\hline
\end{tabular}

20. Que los profesionales me escuchen y que cuenten con mi opinión para planificar el tratamiento y la rehabilitación de mi familiar 
21. Que los profesionales me hablen con palabras que yo pueda comprender y en mi propio idioma

\begin{tabular}{l|l|l|l|l} 
HE SENTIDO ESTA NECESIDAD & HE SENTIDO ESTA NECESIDAD & NO HE SENTIDO \\
Y ME LA HAN SATISFECHO & PERO NO ME LA HAN SATISFECHO & ESTA NECESIDAD
\end{tabular}

22. Que los profesionales consideren el derecho de la familia a no asumir responsabilidades que no le corresponden

\begin{tabular}{|c|c|c|}
\hline HE SENTIDO ESTA NECESIDAD & $\begin{array}{llll}\text { HE } & \text { SENTIDO } & \text { ESTA NECESIDAD }\end{array}$ & NO HE SENTIDO \\
\hline
\end{tabular}

Y ME LA HAN SATISFECHO PERO NO ME LA HAN SATISFECHO ESTA NECESIDAD

23. Recibir ayuda para sustituirme en el cuidado de mi familiar enfermo cuando yo no pueda hacerlo

\begin{tabular}{l|l|lll} 
HE SENTIDO ESTA NECESIDAD & HE SENTIDO ESTA NECESIDAD & NO HE SENTIDO \\
Y ME LA HAN SATISFECHO & PERO NO ME LA HAN SATISFECHO & ESTA NECESIDAD \\
\hline
\end{tabular}

24. Recibir atención especializada, especialmente programas de intervención familiar.

\begin{tabular}{l|l|l|l|}
\hline HE SENTIDO ESTA NECESIDAD & HE SENTIDO ESTA NECESIDAD & NO HE SENTIDO \\
Y ME LA HAN SATISFECHO & PERO NO ME LA HAN SATISFECHO & ESTA NECESIDAD \\
\hline
\end{tabular}

25. Necesidad de sentir la compañía de otras personas que tengan el mismo problema que yo

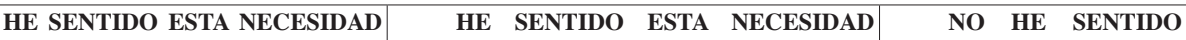
\begin{tabular}{l|l|r|} 
Y ME LA HAN SATISFECHO & PERO NO ME LA HAN SATISFECHO & ESTA NECESIDAD \\
\hline
\end{tabular}

26. Necesidad de sentir la solidaridad de la gente, que me respeten, que me apoyen y que comprendan mi situación 
ORIGINALES Y REVISIONES

27. Necesidad de descansar y de reducir la sensación de responsabilidad en el cuidado de mi familiar enfermo

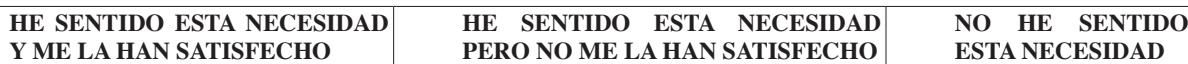

Repase ahora las necesidades que ha sentido pero que no han sido satisfechas ¿Cuál ha sido el motivo por el que no ha podido satisfacerlas? Marque con una cruz uno de los siguientes motivos, aquel que ha sido el más frecuente:

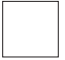

Los profesionales con los que me he relacionado no tienen la costumbre de dar explicaciones a la familia

No he tenido interés en hablar con los profesionales, no creo que puedan aportarme nada útil

$\square$

No he pedido nada a los profesionales ya que ellos están para ayudar solamente a los pacientes y no a los familiares

$\square$

He preguntado a los profesionales pero nunca me han dado explicaciones claras 
ORIGINALES Y REVISIONES

\section{BIBLIOGRAFIA:}

(1) Tennakoon L, Fannon D, Doku V, O'Ceallaigh S, Soni W, Santamaria M, et al. Experience of caregiving: relatives of people experiencing a first episode of psychosis. Br J Psychiatry. 2000 Dec;177:529-33.

(2) Morgan J, Lowe J, Lowe L, Rathod S. An international perspective on what carers want--the professional's dilemma. Int J Soc Psychiatry. 2010 May;56(3):211-3.

(3) Rapaport J, Bellringer S, Pinfold V, Huxley P. Carers and confidentiality in mental health care: considering the role of the carer's assessment: a study of service users', carers' and practitioners' views. Health Soc Care Community. 2006 Jul;14(4):357-65.

(4) Ecob R, Croudace TJ, White IR, Evans JE, Harrison GL, Sharp D, et al. Multilevel investigation of variation in HoNOS ratings by mental health professionals: a naturalistic study of consecutive referrals. Int J Methods Psychiatr Res. 2004;13(3):152-64.

(5) Evans S, Greenhalgh J, Connelly J. Selecting a mental health needs assessment scale: guidance on the critical appraisal of standardized measures. J Eval Clin Pract. 2000 Nov;6(4):379-93.

(6) Tansella CZ. Illness and family functioning: theoretical and practical considerations from the primary care point of view. Fam Pract. 1995 Jun;12(2):214-20.

(7) Thornicroft $\mathrm{G}$. The uses and limitations of mental health service needs indices. Br J Psychiatry. 1996 Dec;169(6):792.

(8) Marshall M, Hogg LI, Gath DH, Lockwood A. The Cardinal Needs Schedule--a modified version of the MRC Needs for Care Assessment Schedule. Psychol Med. 1995 May;25(3):605-17.

(9) Phelan M, Slade M, Thornicroft G, Dunn G, Holloway F, Wykes T, et al. The Camberwell Assessment of Need: the validity and reliability of an instrument to assess the needs of people with severe mental illness. Br J Psychiatry. 1995 Nov;167(5):589-95.

(10) Wennstrom E, Berglund L, Lindback J, Wiesel FA. Deconstructing the 'black box' of the Camberwell assessment of need score in mental health services evaluation. Soc Psychiatry Psychiatr Epidemiol. 2008 Sep;43(9):714-9.

(11) Wennstrom E, Wiesel FA. The Camberwell assessment of need as an outcome measure in routine mental health care. Soc Psychiatry Psychiatr Epidemiol. 2006 Sep;41(9):728-33.

(12) Guinea R. Modelo de atención a las personas con enfermedad mental grave. Colección Documentos IMSERSO. 2007;21005.

(13) Barrowclough C, Marshall M, Lockwood A, Quinn J, Sellwood W. Assessing relatives' needs for psychosocial interventions in schizophrenia: a relatives' version of the Cardinal Needs Schedule (RCNS). Psychol Med. 1998 May;28(3):531-42.

(14) Barrowclough C, Tarrier N, Lewis S, Sellwood W, Mainwaring J, Quinn J, et al. Randomised controlled effectiveness trial of a needs-based psychosocial intervention service for carers of people with schizophrenia. Br J Psychiatry. 1999 Jun;174:505-11.

(15) Drapalski AL, Marshall T, Seybolt D, Medoff D, Peer J, Leith J, et al. Unmet needs of families of adults with mental illness and preferences regarding family services. Psychiatr Serv. 2008 Jun;59(6):655-62.

(16) Roncone R, Mazza M, Ussorio D, Pollice R, Falloon IR, Morosini P, et al. The questionnaire of family functioning: a preliminary validation of a standardized instrument to evaluate psychoeducational family treatments. Community Ment Health J. 2007 Dec;43(6):591-607. 
(17) Morris A, Bloom JR, Kang S. Organizational and individual factors affecting consumer outcomes of care in mental health services. Adm Policy Ment Health. 2007 May;34(3):243-53.

(18) Veltro F, Morosini P, Gigantesco A, Casacchia M, Roncone R, Dell'acqua G, et al. A new self-report questionnaire called "ABC" to evaluate in a clinical practice the aid perceived from services by relatives, needs and family burden of severe mental illness. Clin Pract Epidemiol Ment Health. 2007;3:15.

(19) Magliano L, Fiorillo A, De Rosa C, Malangone C, Maj M. Family burden in long-term diseases: a comparative study in schizophrenia vs. physical disorders. Soc Sci Med. 2005 Jul;61(2):313-22.

(20) Vilaplana M, Ochoa S, Martinez A, Villalta V, Martinez-Leal R, Puigdollers E, et al. Validation in Spanish population of the family objective and subjective burden interview (ECFOS-II) for relatives of patients with schizophrenia. Actas Esp Psiquiatr. 2007 Nov-Dec;35(6):372-81.

(21) Epstein N, Baldwin, L. y Bishop, D. The McMaster family assessment device. J of Marital and Family Therapy. 1883;9:171-80.

(22) Malcolm K RP, Inch H. Assessment Of respite needs for carers of personswith severe mental illness. Psychiatric Bulletin. 1998;22:354-5.

(23) Brand U. European perspectives: a carer's view. Acta Psychiatr Scand Suppl. 2001(410):96101.

(24) Bishop DS, Epstein NB, Baldwin LM. Structuring a family assessment interview. Can Fam Physician. 1980 Nov;26:1534-7.

(25) Wagner LC, King M. Existential needs of people with psychotic disorders in Porto Alegre, Brazil. Br J Psychiatry. 2005 Feb;186:141-5.

(26) Muñoz M, Pérez Santos, E.; Crespo, M.; Guillén, A. Estigma y Enfermedad Mental: Ánalisis del Rechazo Social que Sufren las Personas con Enfermedad Mental. Editorial Complutense: Madrid. 2009.

(27) Guillén A. Evaluación de las Necesidades Psicosociales de Personas con Enfermedad Mental Crónica y de sus Cuidadores Familiares. Editorial Complutense: Madrid. 2010.

(28) Link BG, Struening EL, Neese-Todd S, Asmussen S, Phelan JC. Stigma as a barrier to recovery: The consequences of stigma for the self-esteem of people with mental illnesses. Psychiatr Serv. 2001 Dec;52(12):1621-6.

(29) Lefley HP, Hatfield AB. Helping parental caregivers and mental health consumers cope with parental aging and loss. Psychiatr Serv. 1999 Mar;50(3):369-75.

(30) Smith GC, Hatfield AB, Miller DC. Planning by older mothers for the future care of offspring with serious mental illness. Psychiatr Serv. 2000 Sep;51(9):1162-6.

(31) Poulin MJ, Brown SL, Ubel PA, Smith DM, Jankovic A, Langa KM. Does a helping hand mean a heavy heart? Helping behavior and well-being among spouse caregivers. Psychol Aging. 2010 Mar;25(1):108-17. 